\title{
Analysis on Windows illegal operations
}

\author{
Ying Yu Chen and Hai Dong Jin* \\ School of Computer Science and Technology, Soochow University \\ Suzhou 215006, Jiangsu, China
}

Keyword: Operating system; Windows; GUI; System error; Illegal operations.

\begin{abstract}
Graphical interface for Windows is a multitasking operating system, very easy to use and very broad, but Windows always appear "illegal operation" dialog box. "Illegal operation" operation dialog box has two options: "off" and "details". But the "details" which didn't really point out where problems, makes the most of the users are still bewildered. Below we analyze "illegal operations" details of each of the specific meaning and corresponding solutions.
\end{abstract}

\section{Introduction}

Graphical interface for Windows is a multitasking operating system, very easy to use and very broad, but Windows always appear "illegal operation" dialog box. "Illegal operation" operation dialog box has two options: "off" and "details". But the "details" which didn't really point out where problems, makes the most of the users are still bewildered. Below we analyze "illegal operations" details of each of the specific meaning and corresponding solutions.

\section{Illegal operation}

\section{The stop error number: 0x0000000A.}

Description: IRQL-NOT-LESS-OR-EQUAL

Common causes: driver uses an incorrect memory address.

Solution: If you cannot login, then restart your computer. When the list of available operating system appears, and press the F8 key. On the Windows Advanced Options menu screen, select "last known good configuration" and press ENTER. Check whether any new hardware or software is properly installed. If this is a new installation, contact your hardware or software manufacturer to get any Windows updates or drivers that may be required. Run any system diagnostic software supplied by your computer manufacturer, especially the memory check. Or remove any newly installed hardware (RAM, adapters, hard disks, modems, and so 
on), the driver or the software. Make sure that your hardware device drivers and system BIOS are the latest version. Ensuring that manufacturers can help whether or not you have the latest version, will also give you access to the hardware.

Disable BIOS memory options such as cache or shadow.

\section{The stop error number: 0x0000001E.}

\section{Description: KMODE-EXPTION-NOT-HANDLED}

Common causes: a kernel-mode process attempts to perform an illegal or unknown processor instruction.

Workaround: ensure that sufficient space, especially when we are performing a new installation. If the stop error messages indicate a specific driver, disable him. If you cannot start your computer. Try to boot in safe mode to remove or disable the driver. If you have non-Microsoft supported video drivers, try switching to the standard VGA driver or Windows to provide the appropriate driver. Disable any newly installed drivers. Ensure that the latest version of the system BIOS. Hardware manufacturers can help you determine whether you have the latest version, and can also help you to get him. BIOS memory options such as cache,shadow.

\section{The stop error number: 0x00000023 and 0x00000024.}

Description: FAT-FILE-SYSTEM or MTFS-FILE-SYSTEM

Common cause: the problem occurs in Ntfs. Sys (driver file allows the system to read and write NTFS drives).

Solution: run a system diagnostic software supplied by your computer manufacturer, especially hardware diagnostics.

Disable or uninstall any antivirus software, disk defragmentation or backup programs.

At the command prompt, run the command to check for hard drive corruption, and then restart your computer.

Stop number: 0x0000002E.

Description: DATA-BUS-ERROR

Common causes: System memory parity error, typically caused by hardware problems.

Solution: remove any newly installed hardware (RAM. Adapter. The hard drive. Modems, and so on). Run system diagnostic software supplied by your computer manufacturer, especially hardware diagnostics. Make sure that your hardware device drivers and system BIOS are the latest version. Use the system diagnostics supplied by your hardware vendor, run a memory check to look for faulty or mismatched memory. Disable BIOS memory options such as cache or shadow. After starting when the list of available operating system appears, press F8. On the Windows Advanced Options menu screen, select "Enable VGA mode". And then press 
ENTER. If this does not resolve the problem, you may need to switch to a different video adapter list, list of supported video adapters, see the hardware compatibility list.

\section{Stops number: 0x0000003F.}

Description: NO-MOR-SYSTEM-PTES

Common causes: clean drivers correctly.

Workaround: disable or uninstall any antivirus software, Disk Defragmenter handler or a backup program.

The stop error number: $0 x 00000058$.

Description: FTDISK-INTERN-ERROR

Common causes: a primary drive in a fault-tolerant set fails.

Solution: start the computer by using the Windows installation disc, from the mirror (2nd) boot system drive. About how to edit the Boot. Ini file to point to the mirror image of your system drive Guide, available on the Microsoft support services Web site search for "Edit ARC path".

\section{The stop error number: 0x0000007B.}

\section{Description: INACCESSI-BLE- BOOT-DEVICE}

Common cause: initialize the I/O system (usually refers to the boot device, or file system) failed.

Workaround: boot sector viruses often cause this stop error. Is to use the latest version of antivirus software, check whether the computer has a virus. If a virus is found, you must perform the necessary he should not get removed from your computer, see your antivirus software documentation for information on how to perform these steps. Remove any newly installed hardware (RAM, adapters, modems, and so on). Check the MIcrosoft hardware compatibility list to ensure that all your hardware and drivers are compatible with Windows. If you are using the appropriate SCSI adapter, in addition to obtain the latest WINDOWS driver from the hardware vendor, disable sync negotiation for a SCSI device, check that the SCSI chain is ending, and check the SCSI ID of the device, if you are not sure how to perform these steps, refer to your hardware documentation. If you are using IDE devices, define the on-board IDE port as the only primary port. Check the master/slave/only settings for IDE devices. Remove all IDE devices except hard drive. If you are unable to confirm how they don't look for, you can refer to your hardware documentation. If the computer is formatted with the NTFS file system, you can restart the computer, and then running/r on the system partition command. If the system cannot be started due to an error, use the command console, and run the command. Run a command to determine whether the file system is damaged. If Windows cannot run Chkdsk command, move the drive to another computer running Windows on, and then run the Chkdsk command on the drive on this computer. 


\section{The stop error number: 0x0000007F.}

\section{Description: UNEXPECTED-KERNEL-MODE}

-TRAP

Common causes: is usually caused due to a hardware or software problem, but it is generally caused by a hardware failure.

Solution: check the Microsoft hardware compatibility list to ensure that all your hardware and drivers are compatible with Windows. If this problem is not compatible with the computer's motherboard can produce. Removed by the newly installed hardware. Run any system diagnostic software supplied by your computer manufacturer, especially the memory check. Disable BIOS memory options such as cache or shadow.

\section{The stop error number: $0 \times 00000050$.}

\section{Description: PAGE-FAULT-IN-NONPA GED-A REA}

Common causes: memory error (data cannot be swapped out to disk using the paging file).

Solution: remove any newly installed hardware. Run any system diagnostic software supplied by your computer manufacturer. Especially the memory check. Check if any new hardware or software is properly installed, if this is a new installation, contact your hardware or software manufacturer to get any Windows updates or drivers that may be required. Disable or uninstall any antivirus programs. Disable BIOS memory options such as cache or shadow.

\section{The stop error number: 0x00000077.}

\section{Description: KERNEL-STEL-STACK-INPAGE-ERRO}

Common causes: kernel data from the paging file in the required page is read into memory.

Solution: use the latest version of anti-virus software, check for viruses on your computer. If a virus is found, and then performs the necessary steps to remove him from your computer. See all system diagnostic software provided by the manufacturer, especially the memory check. Disable BIOS memory options such as cache,shadow.

\section{The stop error number: 0x00000079.}

\section{Description: MISMATCHED-HAL}

Common causes: hardware abstraction layer and the kernel or the machine type mismatch (usually occurs in single-processor and multiprocessor configuration files are mixed in the same system).

Workaround: to resolve this error, use the command console to replace the incorrect system files on your computer. Single-processor kernel file system is Ntoskml. Exe, and the multiprocessor kernel file systems is Ntkrnlmp. Exe, however, these files correspond to the file on the installation media; installing Windows2000 
and regardless of what the original file will be renamed to Ntoskrnl. Exe file. HAL file name Hal is also used after Setup. DLL in the installation media, but in the installation media, there are several possible HAL files.

\section{The stop error number: 0xC000021A.}

\section{Description: STATUS-SYSTEM-PR OCESS-TERMINATED.}

Common cause: user mode subsystem, such as Winlogon or the client server runtime subsystem (CSRSS) have been damaged, and could no longer guarantee security.

Solution: remove any newly installed hardware. If you cannot login, then restart your computer. When the list of available operating system appears, press F8. In Windows2000 advanced options menu screen, select: "last known good configuration". Then press the passing. Run recovery console, and allow the system to repair any errors detected.

\section{The stop error number: $0 x \mathbf{C 0 0 0 0 2 2 1 . ~}$}

Description: STATUS-IMAGE-CHECKISU7M - MISMATCH.

Common causes: the driver or system DLL has been corrupted.Workaround: run fault complex control console, and allow the system to repair any errors detected. If, after adding RAM to your computer, error occurs immediately, then the page file may be damaged, or if the new RAM by a faulty or incompatible. Delete Pagefile. Sys and return the system to the original RAM configuration. Run any system diagnostic software supplied by your computer manufacturer, especially the memory check.

\section{Conclusion}

In the operation of Windows XP system is a program that appears illegal operation or an error is encountered by every computer will be. But this time, Windows XP will initiate an error reporting mechanism (Windows Error Reporting, WER for short) that asks the user whether or not to send error information to Microsoft, and the program will stop running.

You can use Windows error reporting to report computer problems to Microsoft. Microsoft uses the problem reports to try to match each problem description and solution. If there are any steps you can take to solve the problem or to find more information using Action Center and Windows will notify you. If there is no solution, then the information sent from a problem report can help Microsoft find or create a new solution. 


\section{Acknowledgements}

Yingyu Chen, student ID Number: 1127404017, currently is an undergraduate student of Computer Science and Technology School of Soochow University. This work was directed by Haidong Jin, haidong @ suda.edu.cn.

\section{Reference}

[1]. Zakariazadeh A, Jadid S, Siano P. Integrated operation of electric vehicles and renewable generation in a smart distribution system[J]. Energy Conversion and Management, 2015:99-110.

[2]. Yin X, Guo S, Hirata $H$, et al. Design and experimental evaluation of a teleoperated haptic robot-assisted catheter operating system[J]. Journal of Intelligent Material Systems and Structures, 2014.

[3]. Dawson R. Engler, M. Frans Kaashoek, James O. Acm Sigops Operating Systems Review,1995

[4]. Yvonne Coady, Gregor Kiczales,Mike Feeley, Norm Hutchinson. Communications of the ACM, 2001

[5]. Lycklama H, L. Bayer D. UNIX Time-Sharing System: The MERT Operating System[J]. Bell System Technical Journal, the, 1978, 57(6):2049-2086.

[6]. E. Grzelakowski M, H. Campbell J, R. Dubman M. The 3B20D Processor \& DMERT Operating System : DMERT Operating System[J]. Bell System Technical Journal, 1983, 62(1):303-322.

[7]. Rasim Alguliev, Fargana Abdullaeva: Illegal Access Detection in the Cloud Computing Environment[J]. Journal of Information Security. 2014(5):65-71

[8]. Oana CRETESCU, Lavinia Roxana LUNGU. Illegal Operations with IT Software and IT Devices. Journal of Applied Business Information Systems[J]. 2012,3(4)

[9]. Salem, M.B. and Stolfo, S.J. Data Collection and Analysis for Masquerade Attack Detection: Challenges and Lessons Learned. Columbia University Computer Science Technical Reports, Columbia University, (2011) ,8p.

[10]. Lane, T. and Brodley, C.E. (1997) An Application of Machine Learning to Anomaly Detection. Proceedings of the 20th National Information Systems Security Conference, 14 February, 366-380.

[11]. M. Ortega-Vazquez, D. Kirschen.Estimating spinning reserve requirements in systems with significant wind power generation penetration. IEEE Trans Power Syst, 24 (2009), pp. 114-124 